\section{OPEN ACCESS}

Edited by:

J. Michelle Kahlenberg, University of Michigan, United States

Reviewed by:

Thomas S. McCormick, Case Western Reserve University,

United States

David D. Roberts,

National Institutes of Health (NIH),

United States

*Correspondence:

Hortensia de la Fuente

hortensia.fuente@salud.madrid.org

tThese authors have contributed equally to this work

Specialty section:

This article was submitted to Autoimmune and Autoinflammatory Disorders,

a section of the journal

Frontiers in Immunology

Received: 25 January 2019 Accepted: 17 May 2019

Published: 04 June 2019

Citation:

Rodríguez-Jiménez $P$, Chicharro $P$

Llamas-Velasco M, Cibrian D,

Trigo-Torres L, Vara A

Jiménez-Fernández $M$

Sevilla-Montero J, Calzada MJ,

Sánchez-Madrid F, de la Fuente $H$ and

Daudén E (2019)

Thrombospondin-1/CD47 Interaction

Regulates Th17 and Treg

Differentiation in Psoriasis.

Front. Immunol. 10:1268.

doi: 10.3389/fimmu.2019.01268

\title{
Thrombospondin-1/CD47 Interaction Regulates Th17 and Treg Differentiation in Psoriasis
}

\section{Pedro Rodríguez-Jiménez ${ }^{1}$, Pablo Chicharro ${ }^{1}$, Mar Llamas-Velasco ${ }^{1}$, Danay Cibrian ${ }^{2}$, Laura Trigo-Torres ${ }^{2}$, Alicia Vara², María Jiménez-Fernández², Javier Sevilla-Montero ${ }^{2}$, Maria J. Calzada ${ }^{2}$, Francisco Sánchez-Madrid ${ }^{2,3}$, Hortensia de la Fuente ${ }^{2,3 *}$ and Esteban Daudén ${ }^{1 \dagger}$}

${ }^{1}$ Department of Dermatology, Instituto de Investigación Sanitaria Princesa, Hospital Universitario la Princesa, Universidad Autónoma de Madrid, Madrid, Spain, ${ }^{2}$ Department of Immunology, Instituto de Investigación Sanitaria Princesa, Hospital Universitario la Princesa, Universidad Autónoma de Madrid, Madrid, Spain, ${ }^{3} \mathrm{CIBER}$ de Enfermedades Cardiovasculares, Institututo de Salud Carlos III, Madrid, Spain

Accumulating evidence on the role of Thrombospondin-1 (TSP-1) in the immune response has emerged during the last years. In spite of the importance of TSP-1 not only as anti-angiogenic factor but also as an immunomodulatory molecule, studies on the role of TSP-1 in psoriasis have been neglected. TSP-1 and CD47 expression were analyzed in skin samples from psoriasis patients and control subjects using RT-PCR and immunofluorescence. Expression of these molecules was also evaluated in peripheral blood CD4+ T cells, moDCs, and circulating primary DCs. The functional role of TSP-1/CD47 signaling axis in psoriasis was assessed in Th17 and Treg differentiation assays. Additionally, small interfering RNA assays specific to TSP-1 were performed in CD4+ $T$ cells and monocyte derived $D C$ to specifically evaluate the function of this protein. Lesional skin of psoriasis patients expressed lower TSP-1 and CD47 mRNA levels compared to non-lesional skin or skin from controls. Immunofluorescence staining revealed decreased expression of CD47 in CD45+ dermal cells from psoriasis samples compared to control subjects. Peripheral CD4+ T cells and circulating primary DCs from psoriasis also expressed lower levels of CD47 compared to controls. Although no significant differences were detected in TSP-1 expression in CD4+ T cells and moDCs between patients and controls, TSP-1 expression in psoriasis patients inversely correlated with disease activity evaluated by the Psoriasis Area and Index Activity. Furthermore, exogenous TSP-1 inhibited Th17 differentiation and stimulated the differentiation of CD4+ T cells toward Treg cells. Furthermore, RNA interference specific for TSP-1 confirmed the role of this molecule as a negative regulator of T cell activation. Because of the impact of TSP-1/CD47 signaling axis in Th17 and Treg differentiation, a dysregulated expression of these molecules in the immune cells from psoriasis patients may favor the exacerbated inflammatory response in this disease.

Keywords: psoriasis, CD47, TSP-1, Th17, Treg cells 


\section{INTRODUCTION}

Psoriasis is considered a chronic inflammatory autoimmune disease characterized by exacerbated proliferation and disturbed keratinocyte maturation, inflammatory dermal infiltrates and changes of the superficial microvasculature that result in an angiogenic phenotype (1). This condition arises from interactions between keratinocytes, infiltrating Interleukin (IL)-17-, IL-22-, and interferon (IFN)- $\gamma$-producing cells, inflammatory macrophages and dendritic cells (DCs) (2). Clinical investigations and experimental studies indicate that IFN- $\gamma$-producing $\mathrm{T}$ helper type (Th) 1 cells and $\mathrm{CD} 4^{+} \mathrm{Th} 17$ cells accompanied by increased expression of IL-17A and accumulation of $\mathrm{CD}^{+}$cytotoxic $\mathrm{T}$ cells in psoriatic lesions cooperate at the interface of innate and adaptive immunity by activating keratinocytes to produce IL-17C. This cytokine together with other keratinocyte-derived mediators sustains chronic inflammation in psoriatic plaques (3-5). Although inflammation mediated by $\mathrm{CD} 4^{+}$Th17 T cells is considered an important trigger in the development of psoriasis, the precise underlying immuno-inflammatory mechanisms are still to be fully defined.

The multicellular protein thrombospondin-1 (TSP-1) is highly considered for its role in vascular health and disease. TSP-1 modulates vascular response and at pathologic levels promotes vascular dysfunction $(6,7)$. TSP-1 regulates multiple cellular events involved in tissue repair including cell adhesion, migration, proliferation, extracellular matrix expression and organization, and regulation of growth factor activity (8). This is possible due to its multi-domain structure which interacts with several cell receptors and matrix proteins (9). Despite TSP-1 is mostly known for its role in modifying the tumor micro-environment through its anti-angiogenic properties (10), growing evidence about the role of TSP-1 in immune response has emerged in the last decade (11-13).

TSP-1 is known to regulate the conversion of tumor growth factor $\beta$ (TGF- $\beta$ ) from latent to an active form (14). On the other hand, TGF- $\beta 1$ is a potent keratinocyte growth inhibitor, and its signaling pathway is downregulated in psoriatic skin leading to abnormal cell proliferation due to a functional decrease in growth regulation $(15,16)$. Independently of TGF- $\beta$ regulation, TSP- 1 may also have suppressive properties by means of its interaction with CD47 in T cells (17). Particularly, in T CD4+ lymphocytes TSP-1/CD47 interaction promotes an anti-proliferative effect as well as the generation of human peripheral regulatory $\mathrm{T}$ (Treg) cells (18-20). Furthermore, when released after the inflammatory response, the interaction between TSP-1 and CD47 reduces inflammation linked to $\mathrm{T}$ cell activation $(20,21)$. Interestingly, methotrexate stimulates the expression of endogenous TSP-1 in primary human $\mathrm{T}$ cells, which could explain its beneficial effects in psoriasis, due to its immunoregulatory effects (22). Human immature monocyte-derived DCs (moDCs) as well are known to spontaneously produce TSP-1, this being enhanced by microbial stimuli (11). It has also been shown that increased TSP-1 levels during DC activation and its interaction with CD47 and CD36, negatively regulate IL-12, tumor necrosis factor $\alpha$ (TNF- $\alpha$ ) and IL-10 release (23-25). Although our knowledge about the functional relevance of TSP-1 in psoriasis is scarce, a defective expression of TSP-1 in keratinocytes from psoriasis patients correlates with a higher angiogenic response (26). In this paper, we sought to the role of TSP-1/CD47 signaling axis in the development and maintenance of psoriatic lesions. Our results showed that TSP-1 binding to its receptor CD47 was able to inhibit the differentiation of Th17 cells and favor differentiation of CD4+ T cells toward Treg cells. Therefore, a diminished expression of TSP-1 and its receptor CD47 in immune cells from psoriasis patients may promote the exacerbated inflammatory response characteristic of this disease.

\section{METHODS}

\section{Patients}

This study was approved by the Institutional Review Board (IRB)/Independent Ethics Committee of Hospital de la Princesa according to the Declaration of Helsinki Principles. After giving informed consent, 20 healthy individuals and 30 untreated psoriatic patients were enrolled. Patients were eligible for the study if they had a Psoriasis Assessment Severity Index (PASI) $\geq 8$. The following washout periods were established: 14 days for topical corticosteroids, 28 days for systemic treatment including corticosteroids, methotrexate, cyclosporine, acitretin, or phototherapy and 3 months for biologic agents. From each psoriasis patient, two non-sun-exposed cutaneous biopsies $(10 \mathrm{~mm})$ were taken, one from lesional psoriatic skin and other from apparently healthy skin (non-lesional skin). At the same time, $20 \mathrm{ml}$ of peripheral venous blood were obtained. Normal leftover skin samples and peripheral venous blood samples were obtained from 20 surgical patients. Each biopsy was cut in half; one piece was snap frozen for RNA isolation and the other one was embedded in OCT $^{\mathrm{TM}}$ compound (Sakura Finetek, Zoeterwoude, NL) and stored at $-80^{\circ} \mathrm{C}$ until processing for immunofluorescence.

\section{Expression of TSP-1 and CD47 mRNA Levels by RT-PCR}

TSP-1 and CD47 mRNA expression levels were determined by reverse transcription polymerase chain reaction (RT-PCR). Total RNA was isolated from skin samples, peripheral blood CD4+ $\mathrm{T}$ cells and moDCs using TRIzol reagent (Invitrogen) following the manufacturer's instructions. One microgram of RNA was reverse-transcribed to cDNA and amplified with the specific primers pairs (TSP-1 forward GCC ACA GTT CCT GAT GGA G, reverse CCA TGG AGA CCA GCC ATC; CD47 forward TCC ACA GCA CAG CCA AGG T, reverse TCG CAG ATG ACT TGA GAG TGA AC) using GoTaq qPCR Master Mix (Promega, WI USA). The data were analyzed using StepOne Plus Software (Applied Biosystems, Carlsbad, CA). TSP-1 and CD47 mRNA levels were normalized to GAPDH levels.

\section{Immunofluorescence Staining}

Skin OCT sections of $5 \mu \mathrm{m}$ were fixed (formaldehyde $4 \%$ ), permeabilized (Triton X-100 0,2\%) and blocked with $100 \mu \mathrm{g} / \mathrm{ml}$ human gammaglobulin (Sigma-Aldrich, St. Louis MO, USA) and a 1:100 dilution of donkey serum (Sigma-Aldrich) in phosphate 
buffer solution (PBS). Skin sections were then incubated overnight with $5 \mu \mathrm{g} / \mathrm{ml}$ sheep anti-human CD47 (R\&D systems, Cat. AF4670) and mouse anti-human CD45, followed by donkey anti-sheep (DAS) Alexa Fluor 488 and donkey anti-mouse (DAM) Alexa Fluor 555. Finally, cell nuclei were counterstained with DAPI. Negative controls were performed with omission of the primary antibody. Sections were examined with a Leica DMR immunofluorescence microscopy under the same acquisition conditions. Images were analyzed using the ImageJ sowftware (http://imagej.softonic.com). For the analysis of CD47 expression, fluorescence intensity was determined in regions of interest (ROIs) drawn on CD45+ cells.

\section{Peripheral Blood CD4+ T Cells and Monocyte Derived DCs (moDCs)}

Peripheral blood mononuclear cells (PBMNCs) were obtained by density gradient centrifugation and then CD4+ $\mathrm{T}$ cells were isolated by negative selection using magnetic microbeads (Miltenyi Biotec, Bergisch Gladbach, Germany). For moDCs, PBMNCs were allowed to adhere for $30 \mathrm{~min}$ at $37^{\circ} \mathrm{C}$, and plastic adhered cells were cultured for 5 days in complete media supplemented with $500 \mathrm{U} / \mathrm{ml} \mathrm{GM-CSF}$ (Peprotech) and $10 \mathrm{ng} / \mathrm{ml}$ IL-4 (R\&D systems). On day $6,10 \mathrm{ng} / \mathrm{ml}$ LPS were added and after $24 \mathrm{~h}$ cells were harvested.

\section{Th17 and Treg Differentiation Assays}

For Th17 differentiation, isolated CD4+ $\mathrm{T}$ cells were cultured for 10 days with anti-CD3 (BioLegend Cat\# 300314, RRID:AB314050) plus anti-CD28 mAbs (BD, Cat\#555725) (at $5 \mu \mathrm{g} / \mathrm{ml}$ and $2 \mu \mathrm{g} / \mathrm{ml}$, respectively) in the presence of the combination of cytokines and blocking antibodies appropriate for polarization: rhIL-6 h IL-1 $\beta$ (10 ng/ml), rhIL-23 $(20 \mathrm{ng} / \mathrm{ml})$, rhTGF- $\beta 1 \quad(2 \mathrm{ng} / \mathrm{ml})$, anti-IFN- $\gamma(10 \mu \mathrm{g} / \mathrm{ml})$ and anti-IL-4 $(10 \mu \mathrm{g} / \mathrm{ml})$. For Treg polarization, cells were cultured with TGF- $\beta(5 \mathrm{ng} / \mathrm{ml})$ and IL-2 $(20 \mathrm{U} / \mathrm{ml})$ (all cytokines from R\&D systems) for 5 days. Where indicated hTSP-1 $(5 \mu \mathrm{g} / \mathrm{ml})$, mouse anti-human CD47 $(1 \mu \mathrm{g} / \mathrm{ml})$ clone B6H12.2 (Abcam Cat\# ab3283, RRID:AB303671) or IgG1 isotype control were also added. Percentage of IL-17+ and FoxP3+ CD25+ cells (Treg) was measured in a FACS Canto cytometer and analyzed with FlowJo software.

\section{siRNA Transfection}

Transfections were carried out using 4-D Nucleofector and P3 primary cell kit (Amaxa Lonza, Köln, Germany). Unstimulated CD4+ $\mathrm{T}$ cells or immature moDCs $\left(1 \times 10^{6}\right.$ cells $)$ were transfected with TSP-1 specific siRNA (50 pmol) selecting the EO-115 protocol from the 4-D Nucleofector for CD4+ $\mathrm{T}$ cells and the protocol CM-120 for moDCs. Immediately after nucleofection, cells were incubated in X-VIVO 15 (Lonza, Belgium) medium.

\section{Mixed Leukocytes Reaction (MLR) Assay}

CD4+ T cells were co-cultured with moDCs from a different donor in 96-well U-bottom plates in X-VIVO 15 medium. The ratio of DCs and CD4+ $\mathrm{T}$ cells was 1:5. CD4+ T cells were preloaded with CellTrace Violet (Invitrogen, by Thermo
Fisher Scientific, OR USA) to follow cell proliferation. Cells were cultured for 5 days, then intracellular cytokine production and CellTrace Violet dilution was assessed by flow cytometry.

\section{Treg Suppression Assays}

After 5 days of differentiation, Treg cells were isolated using magnetic beads and added to MLR cultures (ratio 1:2). Responder CD4+ $\mathrm{T}$ cells were from the same donor of Treg cells. Immediately before the co-culture, responder $\mathrm{T}$ cells were loaded with CellTrace Violet, while Treg cells were loaded with CFSE (Invitrogen). After 5 days of culture, proliferation of responder cells was evaluated by flow cytometry.

\section{Statistical Analysis}

Data were analyzed with GraphPad Prism (GraphPad Software, San Diego, CA, USA). One Way Anova, Tukey test and MannWhitney $U$-test were used as appropriate. Where indicated, Wilcoxon signed rank test was used to paired data. Data from $\mathrm{T}$ cell differentiation assays were analyzed using Friedman test and Dunn's Multiple Comparison. The Spearman test was used for correlation analysis. Differences were considered significant at $p<0.05$.

\section{RESULTS}

Expression of TSP-1 and CD47 was analyzed by RT-PCR in skin samples from psoriasis patients and healthy controls. Our data showed that lesional skin from psoriasis patients express lower levels of TSP-1 and CD47 compared to nonlesional skin or skin from control subjects (Figures 1A,B). Immunofluorescence assays showed that CD47 is expressed in the dermis and epidermis of both control and psoriasis skin samples. Furthermore, double immunostaining with CD45 identified the expression of CD47 in dermal leukocytes (Figure 1C). Quantitative analysis demonstrated diminished levels of CD47 in CD45+ dermal cells of psoriasis patients compared to cells from non-lesional skin or cells from control subjects (Figure 1D). Conversely, we did not observe any difference in the levels of CD47 in keratinocytes between psoriasis patients and healthy controls (Figure 1E).

Expression of CD47 and TSP-1 was also analyzed in peripheral blood CD4+ $\mathrm{T}$ cells and monocyte-derived DCs (moDCs). Our data showed that peripheral CD4+ T cells from psoriasis patients expressed lower levels of CD47 compared to healthy controls (Figure 2A). Although our results did not prove significant differences in TSP-1 mRNA levels between healthy subjects and psoriasis patients (Figure 2A), statistical analysis showed a negative correlation between TSP-1 expression and Psoriasis Assessment Severity Index (PASI) (Figure 2B). However, no correlation between CD47 expression and PASI was observed (Figure 2B).

The expression of TSP-1 and CD47 was also analyzed in moDCs under basal conditions or following activation with LPS. No significant differences were observed in the basal expression of TSP-1 and CD47 between psoriasis patients and healthy controls (Figure 2C). However, similarly to our findings in peripheral CD4 $+\mathrm{T}$ cells, TSP-1 expression levels in 


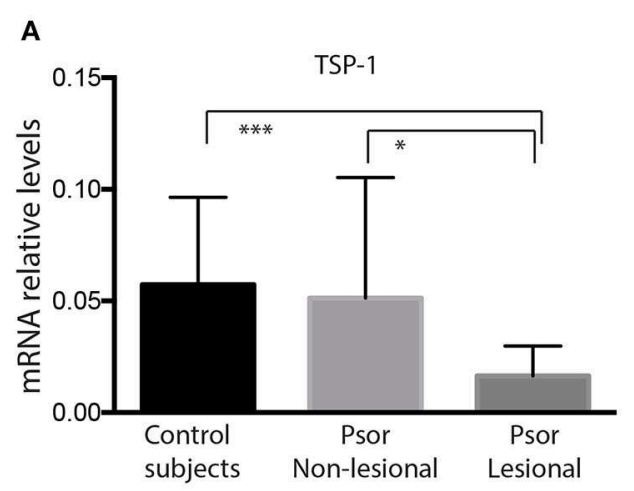

C
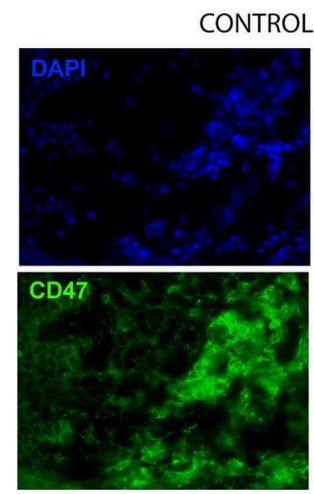

D
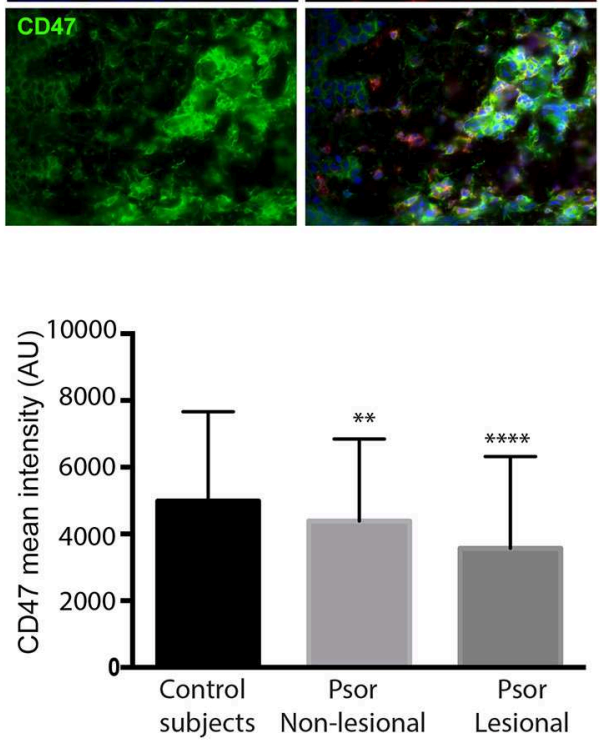

B

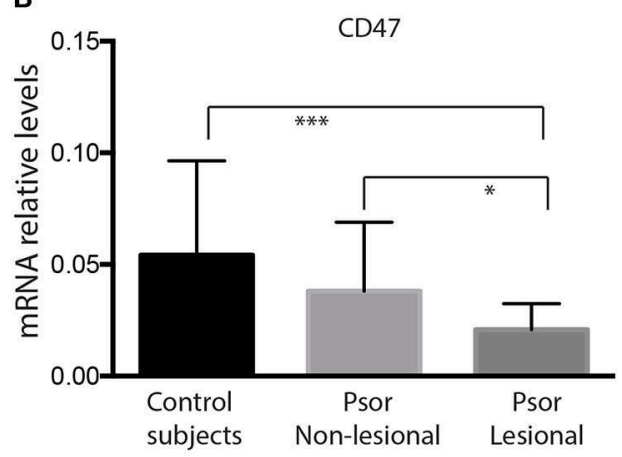

PSORIASIS (LESIONAL SKIN)
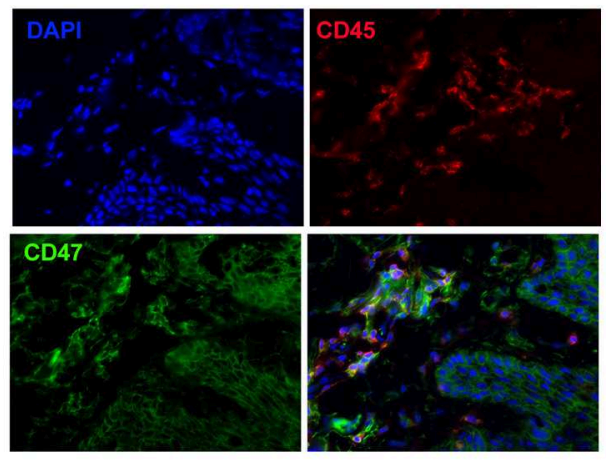

E
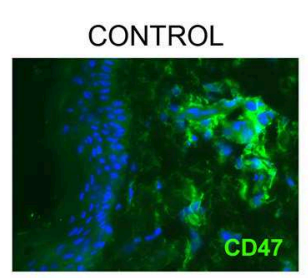

PSORIASIS

(LESIONAL SKIN)

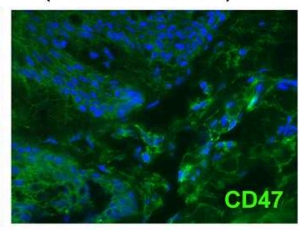

FIGURE 1 | Skin samples from psoriasis patients express lower levels of TSP-1, and CD47 compared with healthy controls. mRNA levels of TSP-1 (A) and its receptor CD47 (B) were analyzed by RT-PCR in skin samples from 26 psoriasis patients and 20 healthy controls. GAPDH expression was used to normalize gene expression. Data were analyzed by one-way ANOVA followed by Tukey's multiple comparisons test, ${ }^{* * *} p<0.001$, ${ }^{*} p<0.05$. (C) Double immunofluorescence staining of CD47 (green) and CD45 (red) in a representative skin sample from control subjects (left panels) and lesional skin from psoriasis patients (right panels) is shown. Nuclei were counterstained with DAPI (blue). (D) For quantification of immunofluorescence staining, fluorescence intensity of CD47 in CD45+ dermal cells was calculated using Image J software. (E) Representative expression of CD47 (green) in skin samples from control subjects and psoriasis patients. Graphs represent mean $\pm \mathrm{SD}$. Differences between groups were determined by one-way ANOVA followed by Tukey's multiple comparisons test, ${ }^{* \star \star \star} p<0.0001,{ }^{\star \star} p \leq 0.001$.

immature moDCs negatively correlated with PASI (Figure 2D). Interestingly, TSP-1 induction in response to LPS was lower in moDCs from psoriasis patients compared to controls, while no significant differences in CD47 expression levels were found (Figure 2E).

CD47 protein levels were evaluated by flow cytometry in plasmacytoid DCs (pDCs) and myeloid DCs (mDCs) from psoriasis patients and healthy controls. DCs were identified as HLA-DR $^{+}$Lineage $^{-}$(CD3, CD14, CD20, CD56) cells and then selected according to CD123 and CD11c expression (pDCs and mDCs, respectively) (Figure 3A). Although CD47 protein levels were high in both pDCs and mDCs, we did not detect any significant difference in the expression of CD47 between both cell types (Figure 3B). However, both subsets of circulating DCs from psoriasis patients expressed significantly lower levels of CD47 compared to cells from healthy subjects (Figures 3B,C).

To evaluate the functional role of CD47 and TSP-1 in psoriasis inflammatory response we performed Th17 and Treg 

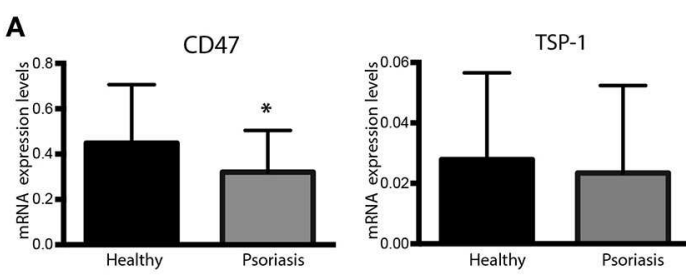

C
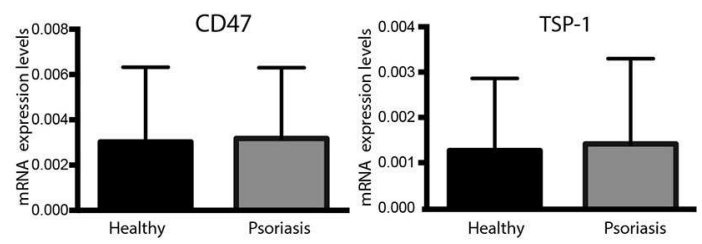

E

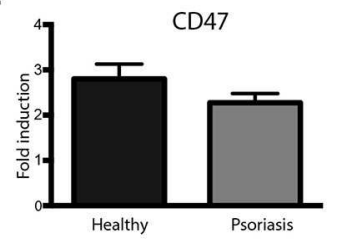

B
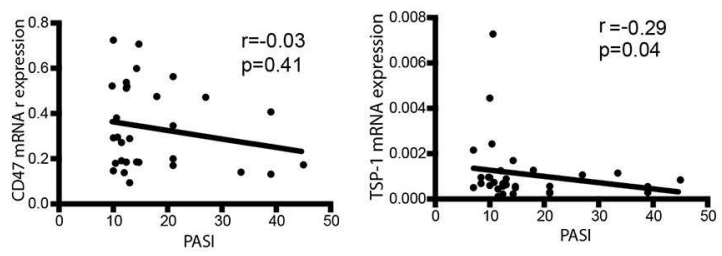

D
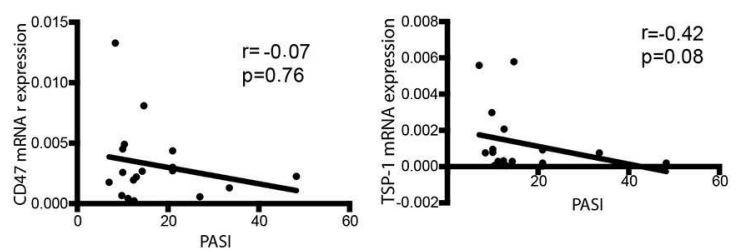

FIGURE 2 | Dysregulated expression of CD47 and TSP-1 in peripheral blood CD4+ T lymphocytes and moDCs. (A,B) CD4+ T cells from psoriasis patients $(n=30)$ and healthy controls $(n=18)$ were isolated from peripheral blood using magnetic microbeads. (A) CD47 and TSP-1 expression was analyzed using real-time PCR. GAPDH expression was used to normalize data. Differences between groups were analyzed using Mann-Whitney $U$-test. (B) Correlation between the expression of CD47 and TSP-1 in CD4+ T cells and PASI determined by Spearman test. (C) Basal expression of CD47 and TSP-1 in non-stimulated moDCS from patients and controls. Monocytes were isolated from peripheral blood using immune-magnetic bead and differentiated to DCs during 5 days in the presence of IL-4 and GM-CSF. (D) Correlation between the expression of CD47 and TSP-1 in moDCs cells and PASI determined by Spearman test. (E) Fold induction of CD47 and TSP-1 expression levels in moDCs in response to LPS. moDCs were stimulated for $24 \mathrm{~h}$ in the presence of LPS (10 ng/ml), then RNA was isolated and the expression of TSP-1 and CD47 analyzed by RT-PCR, calculating their fold induction as LPS/basal expression $(n=18)$. Differences were tested by Mann-Whitney $U$-test, ${ }^{\star} p<0.05$.

differentiation assays on peripheral CD4+ T cells from psoriasis patients and healthy controls. CD4+ T cells were incubated with the corresponding cocktail of cytokines, and in the presence of human TSP-1 or anti-CD47 mAb that mimics TSP-1 binding (20) (Figures 4A,B). Our results demonstrated a clear and significant reduction in the percentage of $\mathrm{IL}-17+$ cells when these cells were cultured in the presence of TSP-1 or anti-CD47 antibody (Figures 4A,C) in psoriasis patients (Friedman test $p=0.003$ ). Similar to our data from psoriasis patients, TSP-1 and antiCD47 reduced the percentage of IL-17+ cells in cell cultures from healthy donors (Friedman test $p=0.007$ ) (Figure 4C). In addition, we assessed whether TSP-1 or anti-CD47 mAb were able to affect Treg differentiation. Our results showed that the addition of TSP-1 induced a significant increment in the percentage of Treg (CD25+ FoxP3+) cells in samples from psoriasis patients (Friedman test $p=0.03$ ) (Figures 4B,D). Although we observed a tendency for anti-CD47 to favor Treg differentiation, differences were not significant (Figure 4D). In order to analyze the functionality of CD25+ FoxP3 + cells, we tested their ability to suppress $\mathrm{T}$ cell proliferation. Our data showed that CD25+ FoxP3 + cells differentiated in the presence of TSP-1 or anti-CD47 mAb were able to inhibit the proliferation of $\mathrm{T}$ cells in a mixed leukocytes reaction (MLR) (Figure 4E). To confirm the role of TSP-1 in CD4+ T cell activation, siRNAmediated silencing was performed. The levels of exogenously expressed TSP-1 were monitored by flow cytometry. As shown in Figure 4F, protein levels dropped in the presence of siRNA targeted to TSP-1. Both CD25 expression and IFN- $\gamma$ production were clearly augmented in T cells transfected with TSP-1-specific siRNA, when they were activated via TCR (Figure 4F).

Finally, the role of TSP-1/CD47 signaling axis was evaluated in antigen (Ag) presentation assays with autologous co-cultures of moDCs with CD4+ T lymphocytes and using the expression of CD25 as a marker of activation. These assays showed that the anti-CD47 mAb clearly prevented the activation of CD4+ $\mathrm{T}$ cells during Ag presentation (Figure 5A) in cells from psoriasis patients. However, the addition of human TSP1 had no significant effects on CD25 expression (Figure 5A). No significant differences were observed in the co-cultures of cells from control subjects (Figure 5A). These findings contrast with those observed in $\mathrm{T}$ cell differentiation and activation (Figure 4). The effect of exogenous TSP-1 could be dose dependent, and it is also important to remark that anti-CD47 (clone B6H12.2) not only mimics TSP-1 binding but blocks as well the binding of SIRP alpha (27) expressed in DCs. Thus, to elucidate whether or not TSP-1 expressed by DCs 
A
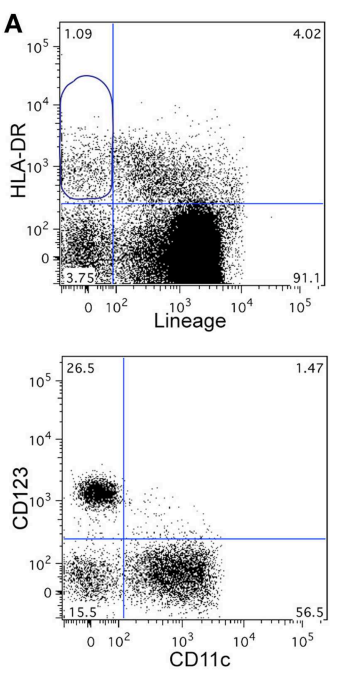

C

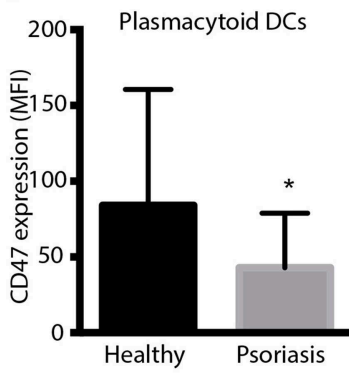

B
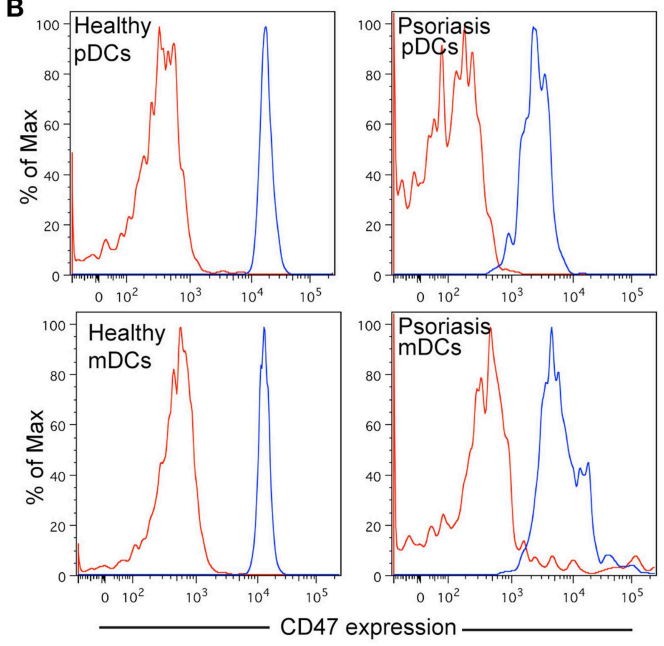

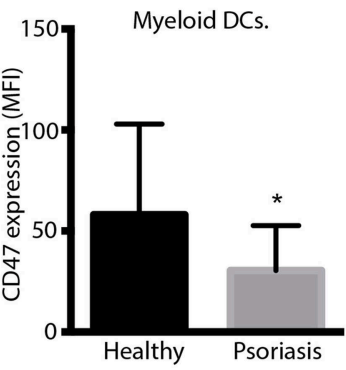

FIGURE 3 | Peripheral blood plasmacytoid and myeloid DCs from psoriasis patients express low levels of CD47. (A) Gating strategy for the detection of pDCs and mDCs. Dendritic cells were identified as HLA-DR positive, Lineage (CD3, CD14, CD20, CD56) negative, CD47 expression was then determined in CD123+ and CD11c+ by flow cytometry. (B) Representative histograms of CD47 expression in pDCs and mDCs from one patient and one control subject. Red and blues lines indicates isotype control and CD47 expression, respectively. (C) CD47 expression was calculated as mean fluorescence intensity (MFI) of CD47/MFI isotype control. Bars represent mean \pm SD from 18 psoriasis patients and 10 control subjects. Differences were analyzed by the Mann-Whitney $U$-test, ${ }^{*} p<0.05$.

was playing a role in $\mathrm{CD} 4+\mathrm{T}$ cell activation, endogenous expression of TSP-1 was silenced in moDCs by siRNA assays. T cell activation in MLR assays, evaluated as CD25 expression, IFN$\gamma$ production and proliferation, was augmented in the presence of moDCs transfected with TSP-1 siRNA (Figure 5B). These data demonstrate that TSP-1 expressed by moDCs is involved in the activation of $\mathrm{CD} 4+\mathrm{T}$ cells.

\section{DISCUSSION}

TSP-1/CD47 signaling axis has been considered an important sensor to maintain homeostasis and regulate innate and adaptive immune responses (28). In this study we identified a defective expression of CD47 not only locally in dermal leukocytes, but also in CD4+ T lymphocytes, $\mathrm{pDCs}$, and $\mathrm{mDC}$ from peripheral blood. The impact of CD47 deficiency in skin inflammation has been studied in animal models of contact hypersensitivity (CHS). An exacerbated T cell-mediated CHS response and prolonged inflammation indicate the importance of CD47 in different phases of the inflammatory response $(21,29)$. Moreover, a role during disease resolution in CHS has been observed in
TSP-1 deficient mice (21). TSP-1 is present in low amounts in almost every tissue, but it is rapidly and transiently increased under stress or in damaged tissues in response to inflammatory signals (30). In healthy human skin, TSP-1 is produced by basal epidermal keratinocytes and is deposited in the dermoepidermal basement membrane zone, contributing to the barrier that prevents the growth of blood vessels into the dermis. On the contrary, TSP-1 expression is downregulated in diseased skin, such as in skin squamous cell carcinomas (31) and in keratinocytes from psoriasis patients (26). T cells, macrophages and DCs may produce and express surface-bound TSP-1 (3234). Our present results confirmed that TSP-1 levels are decreased in the skin of psoriasis patients, although no differences were detected in the expression of TSP-1 in peripheral CD4+ T cells or moDCs between patients and controls, However, we have shown that moDCs from psoriasis patients failed to upregulate TSP-1 expression in response to LPS. This is in agreement with the increase of TSP-1 in TLR-activated DCs vs. immature DCs observed by other authors (11). Thus, TSP1 might be considered an important brake in psoriasis and this could be mediated by its dual effect impairing angiogenesis 
A
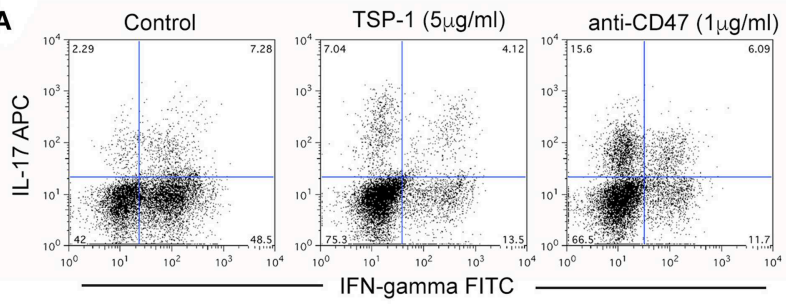

B
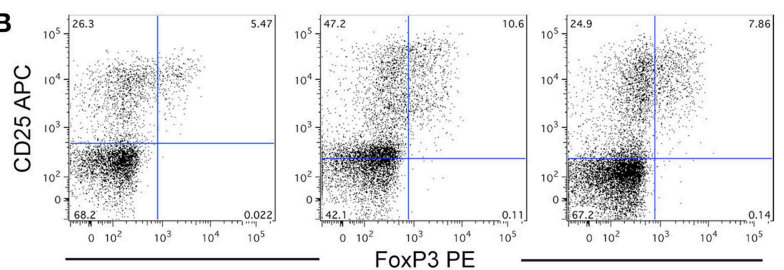

C
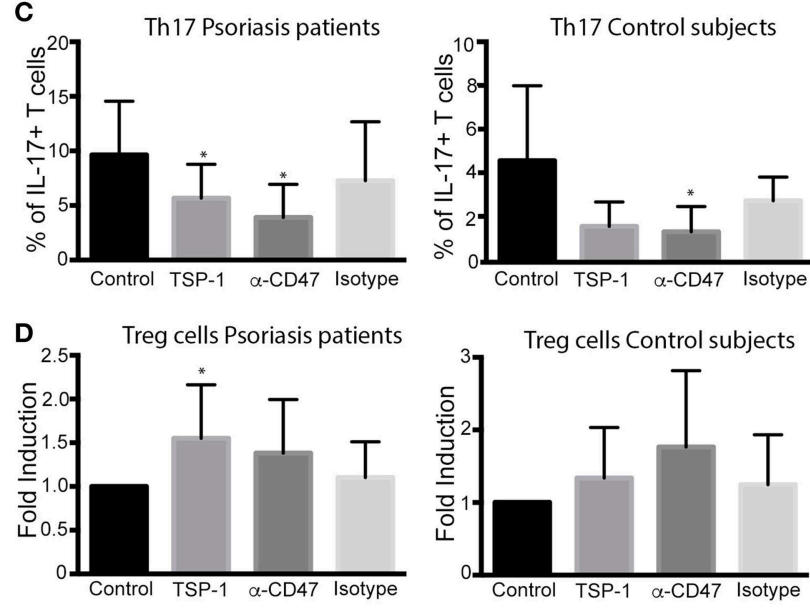

E

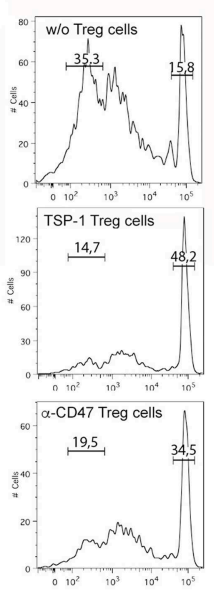

$\mathbf{F}$
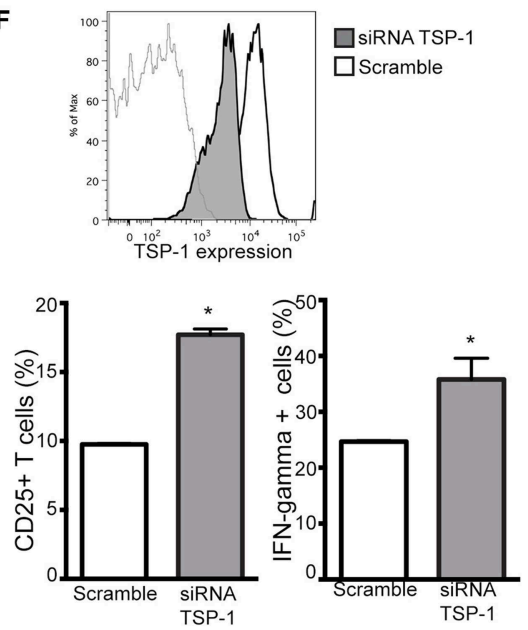

FIGURE 4 | Functional effects of TSP-1 in CD4+ T lymphocytes from psoriasis patients. (A,B) Representative dot plots from Th17 (A) and Treg differentiation (B) assays in the presence of TSP-1 and anti-CD47 mAb. CD4+ T cells were isolated from peripheral venous blood from psoriasis patients using magnetic beads. Cells (1 $\times 10^{6} / \mathrm{ml}$ ) were differentiated to Th17 in the presence or absence of human anti-CD47 mAb $1 \mu \mathrm{g} / \mathrm{ml}$ (left) or human TSP-1 $5 \mu \mathrm{g} / \mathrm{ml}$ (center). (C) Percent of IL- $17+\mathrm{T}$ cells from psoriasis patients $(n=13)$ and control subjects $(n=5)$ evaluated by flow cytometry after 10 days of culture in the presence of TSP-1, anti-CD47 mAb, or lgG1 isotype control $(1 \mu \mathrm{g} / \mathrm{ml})$. Data were analyzed using Friedman test and Dunn's multiple comparison test, ${ }^{*} p<0.05$. (D) Differentiation of Treg cells in the presence of anti-CD47 mAb (1 $\mu \mathrm{g} / \mathrm{ml})$, TSP-1 $(5 \mu \mathrm{g} / \mathrm{ml})$ or lgG1 isotype control $(1 \mu \mathrm{g} / \mathrm{ml})$ from psoriasis patients $(n=9)$ and control subjects $(n=6)$. Graphs show the fold induction of FoxP3+ CD25+ cells in the presence of the indicated stimuli compared to the cells cultured only with the differentiation cocktail. (E) Treg suppression assay, Treg cells differentiated in the presence of TSP-1 or anti-CD47 mAb were isolated by immunomagnetic beads and added to mixed leukocytes reaction (MLR) cultures. Upper histogram corresponds to control culture (moDCs plus responder T cells), central and lower histograms correspond to MLR cultures plus Treg cells that were differentiated in the presence of TSP-1 or anti-CD47 mAb (histograms are representative of two independent experiments). (F) Knockdown of TSP-1 favors T cell activation via TCR. Histogram showing the knockdown efficiency of TSP-1 siRNA. Briefly, unstimulated CD4+ T cells were transfected by nucleofection with TSP-1 specific or control siRNA (50 pmol), $24 \mathrm{~h}$ later T cells were stimulated with anti-CD3/CD28 + IL-2 during $48 \mathrm{~h}$. CD25 expression and IFN- $\gamma$ production were evaluated by flow cytometry data. Data correspond to one out of two independent experiments performed by triplicate. Differences were tested by Mann-Whitney test, ${ }^{*} p<0.05$.

in the skin and regulating Th17 and Treg cell differentiation. Results from in vitro experiments have previously demonstrated that exogenously added TSP-1 or CD47-binding TSP-1 peptide inhibit IL-12 secretion by monocytes in response to $\mathrm{T}$ celldependent stimuli (35). Moreover, TSP-1 co-stimulates TCRactivated $\mathrm{T}$ cells through its interaction with $\alpha 4 \beta 1$ integrin (17). Our data demonstrate that TSP-1-CD47 interaction or the incubation with anti-CD47 $\mathrm{mAb}$ inhibits the differentiation of Th17 cells and favors CD4+ $\mathrm{T}$ cells differentiation into Treg cells from psoriasis patients. Topical administration of a CD47-binding TSP-1 peptide during the development of an ocular inflammation has been described to attenuate clinical symptoms of Sjögren syndrome-associated dry eye and augment FoxP3 expression (36). This is in agreement with previous results suggesting that TSP-1-mediated activation of TGF- $\beta$, as well as its binding to CD47 may create an anti-inflammatory environment in certain immune privileged sites such as the eye (37). It is well-established that CD47/SIRP alpha interaction negatively regulates antigen presentation (27). The anti-CD47 (clone B6H12.2) used in our functional assays, has been described 

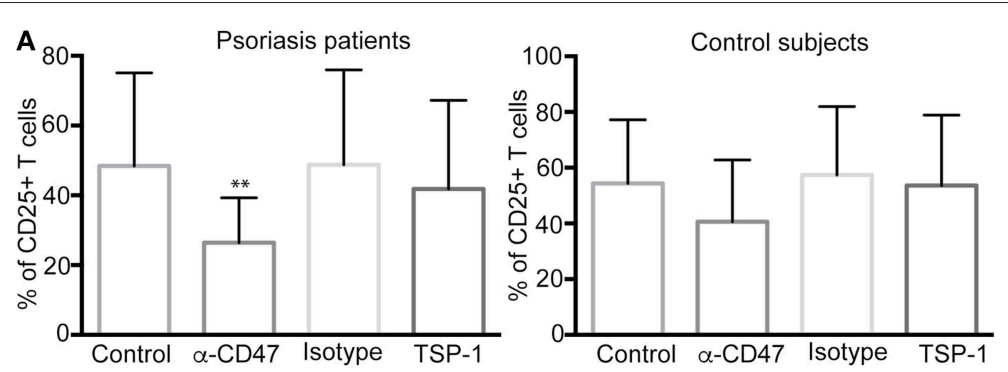

B
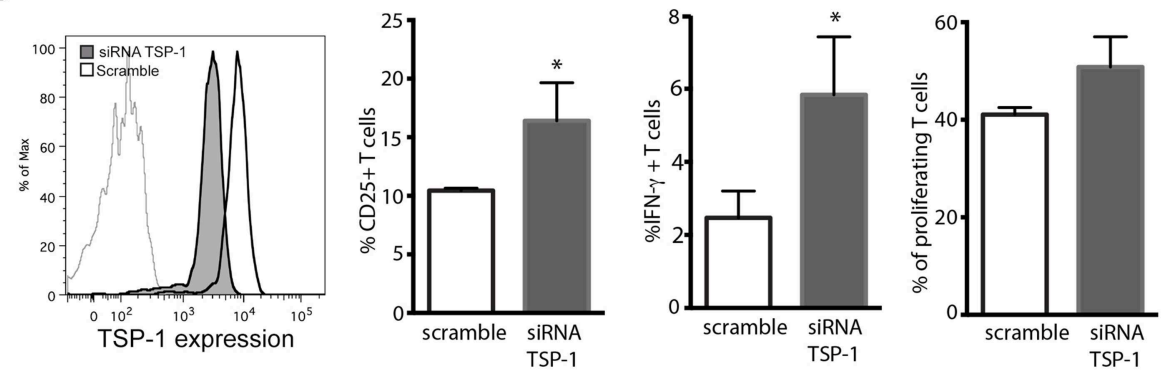

FIGURE 5 | CD47/TSP-1 signaling prevents CD4+ T cell activation in an antigen presentation assay. (A) CD25 expression from CD4+ T cells after antigen presentation, monocyte-derived DCs from psoriasis patients $(n=8)$ and healthy controls $(n=3)$ were cultured for 3 days with autologous peripheral blood CD4+ T cells in the presence of staphylococcal enterotoxin E. Where indicated, anti-CD47 (1 $\mu \mathrm{g} / \mathrm{ml})$, lgG1 isotype control ( $1 \mu \mathrm{g} / \mathrm{ml})$, or TSP-1 (5 $\mu \mathrm{g} / \mathrm{ml})$ were added. Then, expression of CD25 was evaluated using flow cytometry. Data correspond to mean $\pm \mathrm{SD}$ and differences were tested using Friedman test, ${ }^{* *} p<0.01$.

(B) Knockdown of TSP-1 of moDCs augments the activation of T cells following Ag presentation. Histogram showing the knockdown efficiency of TSP-1 siRNA. Briefly, immature moDCs were transfected by nucleofection with TSP-1 specific or control siRNA ( $50 \mathrm{pmol})$, after $48 \mathrm{~h}$ moDCs were stimulated with LPS (10 ng/ml) during $12 \mathrm{~h}$ and then co-cultured with heterologous CD4+ T cells. Data correspond to one out of three experiments performed by triplicate. Differences were analyzed using Mann-Whitney test, ${ }^{*} p<0.05$.

to mimic TSP-1 binding but to also block the binding of CD47 with SIRP alpha. We cannot rule out that the blockade of CD47/SIRP alpha interaction is involved in the effects observed with anti-CD47 mAb. However, in line to previously published data this blockade would lead to a higher $\mathrm{T}$ cell activation instead of the inhibition detected in our assays $(25,27,38)$. In the case of effects of exogenous TSP-1, it is difficult to reproduce the physiological concentration in that particular context. However, TSP-1 silencing assays clearly show that TSP-1 expressed by DCs acts as a negative regulator of immune response.

Together these data suggest that TSP-1 and its receptor CD47 may have a role in the exacerbated inflammatory response characteristic of psoriasis. The link between these molecules and the angiogenesis and immune regulation raises the possibility that they could be evaluated as activity markers in psoriasis.

\section{ETHICS STATEMENT}

Comité de Ética de La investigación con Medicamentos del Hospital Universitario de la Princesa. All patients signed and Informed Consent

\section{AUTHOR CONTRIBUTIONS}

PR-J: acquisition and analysis data and drafting the manuscript. PC: acquisition data and drafting the manuscript. ML-V: analysis and drafting the manuscript. DC: design experiments. LT-T and AV: acquisition data. MJ-F and JS-M: acquisition data, prepared figures and interpreted result. MJC: drafting the article and interpretation of data. FS-M: drafting the article and data interpretation. HdF and ED: design of the work, interpretation, critical revision of the manuscript, and final approval.

\section{FUNDING}

Instituto de Salud Carlos III (AES 2017): PI17/01972 to ED. Janssen; Spanish Ministry of Economy and Competitiveness (MINECO): Plan Nacional de Salud SAF2017-82886-R, Centro de Investigación Biomédica en Red de Enfermedades Cardiovasculares (CIBERCV); Proyecto Integrado de Excelencia PIE13/00041, Instituto de Salud Carlos III to FS-M, Instituto de Salud Carlos III PI16/02166, Universidad Autónoma de MadridBanco Santander (grant 2017/EEUU/03), and Red Temática de Excelencia en Investigación en Hipoxia (SAF 2017-90794-REDT) to MJC. This research has been co-financed by Fondo Europeo de Desarrollo Regional (FEDER).

\section{ACKNOWLEDGMENTS}

We would like to thank Dr. Manuel Gomez Gutierrez for critical reading of the manuscript. 


\section{REFERENCES}

1. Perera GK, Di Meglio P, Nestle FO. Psoriasis. Annu Rev Pathol. (2012) 7:385-422. doi: 10.1146/annurev-pathol-011811-1 32448

2. Hawkes JE, Chan TC, Krueger JG. Psoriasis pathogenesis and the development of novel targeted immune therapies. J Allergy Clin Immunol. (2017) 140:64553. doi: 10.1016/j.jaci.2017.07.004

3. Lowes MA, Kikuchi T, Fuentes-Duculan J, Cardinale I, Zaba LC, Haider AS, et al. Psoriasis vulgaris lesions contain discrete populations of Th1 and Th17 T cells. J Invest Dermatol. (2008) 128:1207-11. doi: 10.1038/sj.jid.57 01213

4. Kryczek I, Bruce AT, Gudjonsson JE, Johnston A, Aphale A, Vatan L, et al. Induction of IL-17+ $\mathrm{T}$ cell trafficking and development by IFNgamma: mechanism and pathological relevance in psoriasis. I Immunol. (2008) 181:4733-41. doi: 10.4049/jimmunol.181.7.4733

5. Res PC, Piskin G, de Boer OJ, van der Loos CM, Teeling P, Bos JD, et al. Overrepresentation of IL-17A and IL-22 producing CD8T cells in lesional skin suggests their involvement in the pathogenesis of psoriasis. PLoS ONE. (2010) 5:e14108. doi: 10.1371/journal.pone.00 14108

6. Isenberg JS, Hyodo F, Pappan LK, Abu-Asab M, Tsokos M, Krishna MC, et al. Blocking thrombospondin-1/CD47 signaling alleviates deleterious effects of aging on tissue responses to ischemia. Arterioscler Thromb Vasc Biol. (2007) 27:2582-8. doi: 10.1161/ATVBAHA.107.155390

7. Labrousse-Arias D, Martinez-Ruiz A, Calzada MJ. Hypoxia and redox signaling on extracellular matrix remodeling: from mechanisms to pathological implications. Antioxid Redox Signal. (2017) 27:802-22. doi: 10.1089/ars.2017.7275

8. Adams JC, Lawler J. The thrombospondins. Cold Spring Harbor Perspect Bioly. (2011) 3:a009712. doi: 10.1101/cshperspect.a009712

9. Calzada MJ, Roberts DD. Novel integrin antagonists derived from thrombospondins. Curr Pharm Design. (2005) 11:849-66. doi: 10.2174/1381612053381792

10. Lawler J. Thrombospondin-1 as an endogenous inhibitor of angiogenesis and tumor growth. J Cell Mol Med. (2002) 6:1-12. doi: 10.1111/j.1582-4934.2002.tb00307.x

11. Doyen V, Rubio M, Braun D, Nakajima T, Abe J, Saito H, et al. Thrombospondin 1 is an autocrine negative regulator of human dendritic cell activation. J Exp Med. (2003) 198:1277-83. doi: 10.1084/jem.20 030705

12. Derks RA, Jankowska-Gan E, Xu Q, Burlingham WJ. Dendritic cell type determines the mechanism of bystander suppression by adaptive $\mathrm{T}$ regulatory cells specific for the minor antigen HA-1. J Immunol. (2007) 179:3443-51. doi: 10.4049/jimmunol.179.6.3443

13. Smith RE, Reyes NJ, Khandelwal P, Schlereth SL, Lee HS, Masli S, et al. Secondary allergic $\mathrm{T}$ cell responses are regulated by dendritic cell-derived thrombospondin-1 in the setting of allergic eye disease. J Leuk Biol. (2016) 100:371-80. doi: 10.1189/jlb.3A0815-357RR

14. Crawford SE, Stellmach V, Murphy-Ullrich JE, Ribeiro SM, Lawler J, Hynes RO, et al. Thrombospondin-1 is a major activator of TGF-betal in vivo. Cell. (1998) 93:1159-70. doi: 10.1016/S0092-8674(00)81460-9

15. Li AG, Wang D, Feng XH, Wang XJ. Latent TGFbetal overexpression in keratinocytes results in a severe psoriasis-like skin disorder. EMBO J. (2004) 23:1770-81. doi: 10.1038/sj.emboj.7600183

16. Han G, Williams CA, Salter K, Garl PJ, Li AG, Wang XJ. A role for TGFbeta signaling in the pathogenesis of psoriasis. J Invest Dermatol. (2010) 130:371-7. doi: $10.1038 /$ jid.2009.252

17. Li Z, Calzada MJ, Sipes JM, Cashel JA, Krutzsch HC, Annis DS, et al. Interactions of thrombospondins with alpha4betal integrin and CD47 differentially modulate $\mathrm{T}$ cell behavior. J Cell Biol. (2002) 157:509-19. doi: $10.1083 /$ jcb. 200109098

18. Wan X, Pei W, Shahzad KA, Zhang L, Song S, Jin X, et al. A tolerogenic artificial APC durably ameliorates experimental autoimmune encephalomyelitis by directly and selectively modulating myelin peptideautoreactive CD4(+) and CD8(+) T cells. J Immunol. (2018) 201:1194-210. doi: $10.4049 /$ jimmunol.1800108
19. Van VQ, Darwiche J, Raymond M, Lesage S, Bouguermouh S, Rubio $\mathrm{M}$, et al. Cutting edge: CD47 controls the in vivo proliferation and homeostasis of peripheral CD4+ CD25+ Foxp3+ regulatory $\mathrm{T}$ cells that express CD103. J Immunol. (2008) 181:5204-8. doi: 10.4049/jimmunol.181. 8.5204

20. Grimbert P, Bouguermouh S, Baba N, Nakajima T, Allakhverdi Z, Braun $\mathrm{D}$, et al. Thrombospondin/CD47 interaction: a pathway to generate regulatory $\mathrm{T}$ cells from human $\mathrm{CD} 4+\mathrm{CD} 25-\mathrm{T}$ cells in response to inflammation. J Immunol. (2006) 177:3534-41. doi: 10.4049/jimmunol.177. 6.3534

21. Lamy L, Foussat A, Brown EJ, Bornstein P, Ticchioni M, Bernard A. Interactions between CD47 and thrombospondin reduce inflammation. J Immunol. (2007) 178:5930-9. doi: 10.4049/jimmunol.178.9.5930

22. Talme T, Bergdahl E, Sundqvist KG. Methotrexate and its therapeutic antagonists caffeine and theophylline, target a motogenic T-cell mechanism driven by thrombospondin-1 (TSP-1). Eur J Immunol. (2016) 46:1279-90. doi: 10.1002/eji.201546122

23. Avice MN, Rubio M, Sergerie M, Delespesse G, Sarfati M. CD47 ligation selectively inhibits the development of human naive $\mathrm{T}$ cells into Th1 effectors. J Immunol. (2000) 165:4624-31. doi: 10.4049/jimmunol.165. 8.4624

24. Johansson U, Londei M. Ligation of CD47 during monocyte differentiation into dendritic cells results in reduced capacity for interleukin-12 production. Scand J Immunol. (2004) 59:50-7. doi: 10.1111/j.0300-9475.2004.0 1354.x

25. Baba N, Van VQ, Wakahara K, Rubio M, Fortin G, Panzini B, et al. CD47 fusion protein targets CD172a + cells in Crohn's disease and dampens the production of IL-1beta and TNF. J Exp Med. (2013) 210:1251-63. doi: 10.1084/jem.20122037

26. Nickoloff BJ, Mitra RS, Varani J, Dixit VM, Polverini PJ. Aberrant production of interleukin- 8 and thrombospondin-1 by psoriatic keratinocytes mediates angiogenesis. Am J Pathol. (1994) 144:820-8.

27. Latour S, Tanaka H, Demeure C, Mateo V, Rubio M, Brown EJ, et al. Bidirectional negative regulation of human $\mathrm{T}$ and dendritic cells by CD47 and its cognate receptor signal-regulator protein-alpha: down-regulation of IL-12 responsiveness and inhibition of dendritic cell activation. J Immunol. (2001) 167:2547-54. doi: 10.4049/jimmunol.167.5.2547

28. Sarfati M, Fortin G, Raymond M, Susin S. CD47 in the immune response: role of thrombospondin and SIRP-alpha reverse signaling. Curr Drug Targets. (2008) 9:842-50. doi: 10.2174/138945008785909310

29. Bouguermouh S, Van VQ, Martel J, Gautier P, Rubio M, Sarfati M. CD47 expression on $\mathrm{T}$ cell is a self-control negative regulator of type 1 immune response. J Immunol. (2008) 180:8073-82. doi: 10.4049/jimmunol.180. 12.8073

30. Raugi GJ, Olerud JE, Gown AM. Thrombospondin in early human wound tissue. J Invest Dermatol. (1987) 89:551-4. doi: 10.1111/1523-1747.ep12461198

31. Streit M, Velasco P, Brown LF, Skobe M, Richard L, Riccardi L, et al. Overexpression of thrombospondin-1 decreases angiogenesis and inhibits the growth of human cutaneous squamous cell carcinomas. Am J Pathol. (1999) 155:441-52. doi: 10.1016/S0002-9440(10)6 5140-1

32. Li SS, Ivanoff A, Bergstrom SE, Sandstrom A, Christensson B, van Nerven $\mathrm{J}$, et al. T lymphocyte expression of thrombospondin-1 and adhesion to extracellular matrix components. Eur J Immunol. (2002) 32:1069-79. doi: 10.1002/1521-4141(200204)32:4<1069::AID-IMMU1069>3.3.CO;2-5

33. Marteau F, Gonzalez NS, Communi D, Goldman M, Boeynaems JM, Communi D. Thrombospondin-1 and indoleamine 2,3-dioxygenase are major targets of extracellular ATP in human dendritic cells. Blood. (2005) 106:38606. doi: 10.1182/blood-2005-05-1843

34. Masli S, Turpie B, Hecker KH, Streilein JW. Expression of thrombospondin in TGFbeta-treated APCs and its relevance to their immune deviation-promoting properties. J Immunol. (2002) 168:2264-73. doi: 10.4049/jimmunol.168.5.2264

35. Armant M, Avice MN, Hermann P, Rubio M, Kiniwa M, Delespesse G, et al. CD47 ligation selectively downregulates human interleukin 12 production. $J$ Exp Med. (1999) 190:1175-82. doi: 10.1084/jem.190.8.1175 
36. Contreras Ruiz L, Mir FA, Turpie B, Masli S. Thrombospondinderived peptide attenuates Sjogren's syndrome-associated ocular surface inflammation in mice. Clin Exp Immunol. (2017) 188:86-95. doi: $10.1111 /$ cei.12919

37. Streilein JW, Masli S, Takeuchi M, Kezuka T. The eye's view of antigen presentation. Hum Immunol. (2002) 63:435-43. doi: 10.1016/S0198-8859(02)0 0393-2

38. Sun FJ, Zhang CQ, Chen X, Wei YJ, Li S, Liu SY, et al. Downregulation of CD47 and CD200 in patients with focal cortical dysplasia type IIb and tuberous sclerosis complex. J Neuroinflamm. (2016) 13:85. doi: 10.1186/s12974-016-0546-2
Conflict of Interest Statement: The authors declare that the research was conducted in the absence of any commercial or financial relationships that could be construed as a potential conflict of interest.

Copyright (C) 2019 Rodríguez-Jiménez, Chicharro, Llamas-Velasco, Cibrian, TrigoTorres, Vara, Jiménez-Fernández, Sevilla-Montero, Calzada, Sánchez-Madrid, de la Fuente and Daudén. This is an open-access article distributed under the terms of the Creative Commons Attribution License (CC BY). The use, distribution or reproduction in other forums is permitted, provided the original author(s) and the copyright owner(s) are credited and that the original publication in this journal is cited, in accordance with accepted academic practice. No use, distribution or reproduction is permitted which does not comply with these terms. 